Matthew J. Hilton · Jacob M. Sawyer • Laura Gutiérrez Amber Hogart • Ting C. Kung • Dan E. Wells

\title{
Analysis of novel and recurrent mutations responsible for the tricho-rhino-phalangeal syndromes
}

\begin{abstract}
The tricho-rhino-phalangeal syndromes (TRPS type I, II, and III) are autosomal dominant disorders sharing the following characteristics: slowly growing and sparse scalp hair, medially thick and laterally thin eyebrows, bulbous tip of the nose, long flat philtrum, thin upper lip with vermilion border, and protruding ears. In addition, individuals with TRPS generally share skeletal and bone anomalies, including shortening of the phalanges and metacarpals (mild to severe brachydactyly), cone-shaped epiphyses, hip dysplasia, and short stature. The etiology of the different types of TRPS can result from either single base pair mutations, or the complete deletion of the TRPS1 gene, which encodes a zinc-finger transcription factor located on chromosomal band 8q24.1. We have identified nine heterozygous mutations, five novel and four recurrent, in unrelated families diagnosed with TRPS. The five novel mutations identified show 1- or 2-bp deletions and a single base substitution, whereas all of the recurrent mutations are single base substitutions. Seven of the nine mutations result in a premature stop codon, leading to a truncated, nonfunctional TRPS1 protein. The final two mutations are missense mutations in the GATA DNA binding zinc finger, which is believed to be important for the protein's normal function.
\end{abstract}

Key words Tricho-rhino-phalangeal syndrome $\cdot$ Mutation Zinc finger $\cdot$ Ikaros $\cdot$ GATA

M.J. Hilton · J.M. Sawyer · L. Gutiérrez · A. Hogart · T.C. Kung · D.E. Wells $(\square)$

Department of Biology \& Biochemistry and Institute for Molecular Biology, University of Houston, 369 Science and Research Bldg 2, Houston, TX 77204-5001, USA

Tel. +1-713-743-2671; Fax +1-713-743-2632

e-mail:dwells@uh.edu

The first two authors contributed equally to this work.

\section{Introduction}

Tricho-rhino-phalangeal syndrome type I (TRPS I, OMIM 190350), type II (TRPS II, OMIM 150230), and type III (TRPS III, OMIM 190351) are autosomal dominant disorders sharing the following characteristics: slowly growing and sparse scalp hair, medially thick and laterally thin eyebrows, bulbous tip of the nose, long flat philtrum, thin upper lip with vermilion border, and protruding ears. In addition, individuals with TRPS generally share skeletal and bone anomalies, including shortening of the phalanges and metacarpals (mild to severe brachydactyly), cone-shaped epiphyses, hip dysplasia, and short stature.

Recently, the TRPS1 gene was identified and mapped to chromosomal band $8 \mathrm{q} 24.1$ by Momeni et al. (2000). The TRPS1 gene appears to encode a zinc-finger transcription factor, which is located proximal to the EXT1 tumor suppressor gene. The contiguous gene syndrome whose deletions encompass both the EXT1 and TRPS1 genes is called Langer-Giedion syndrome (LGS) or TRPS II (Hou et al. 1995; Lüdecke et al. 1995). TRPS II shares the characteristic features of TRPS I with the addition of multiple exostoses, whereas TRPS III only differs clinically from TRPS I by the appearance of severe brachydactyly and extreme shortness of stature (Niikawa and Kamei 1986). Lüdecke et al. (2001) has suggested that TRPS III is a subclass of TRPS I, which specifically contains a missense mutation in the GATA DNA-binding zinc finger of the TRPS1 protein.

We report here the analysis of nine unrelated TRPS families revealing both novel and recurrent heterozygous mutations in the TRPS1 gene. Seven of the nine mutations identified result in a nonfunctional, truncated TRPS1 protein, leading to the typical characteristics found in TRPS I patients. The final two mutations were identified as missense mutations in the GATA DNA-binding zinc finger found in families displaying the characteristic severe brachydactyly and extreme short stature of TRPS III. 


\section{Patients and methods}

Patient DNA preparations. Genomic DNA was isolated from whole blood or buccal samples obtained from TRPS families. Samples were obtained from both normal and affected individuals in each family. DNA isolations were performed using the Puregene DNA isolation kit (D5000) (Gentra Systems, Inc., Minneapolis, MN, USA) according to the manufacturer's protocol.

Polymease chain reaction (PCR) and sequence analysis. PCR was performed in 50- $\mu$ l reactions containing $200 \mu \mathrm{M}$ deoxyribonucleoside triphosphates, $0.5 \mu \mathrm{M}$ TRPS1 primers, $1.5 \mathrm{mM} \mathrm{MgCl}, 1 \times$ Thermophylic buffer, $3.35 \mathrm{U}$ Taq polymerase, and 50-200ng patient DNA using standard PCR conditions. PCR products were cleaned with exonuclease 1 and shrimp alkaline phosphatase (USB, Cleveland, OH, USA) prior to sequencing. Proper concentrations were determined and samples were sequenced using an Applied Biosystems (Foster City, CA, USA) DNA sequencer. PCR products were generated and sequenced with primers described in Momeni et al. (2000). Coding exons (3-7) were sequenced for affected individuals and unaffected individuals from each family.

TRPS patients. Each member of the TRPS families were required to give informed consent prior to their inclusion in this study. TRPS families were referred to our laboratory for deletion analysis and mutation screening of the TRPS1 gene, following a diagnosis of TRPS by the individual's physician or clinician. All individuals surveyed revealed no deletion of the TRPS1 gene (as would be expected in TRPS II individuals), but did exhibit a variety of base substitutions and frameshift mutations within the gene. Each of the affected individuals displayed the characteristic phenotypes of TRPS I, with the exception of families T0153 and T0202. Affected members of these families appeared to have features more indicative of TRPS III. Each of the affected T0153 and T0202 family members suffered from severe brachydactyly as well as a more extreme shortness of stature than that seen in comparable TRPS I patients. Also of interest is the observation that the affected individuals of families T0198 and T0197 suffer from mild learning disabilities and severe clinical depression, respectively.

\section{Results}

Mutation screening of TRPS patients was achieved by PCR amplification and direct sequencing of DNA samples from unaffected and affected individuals from each of the nine families. Our analysis of these families detected five novel and four recurrent mutations. Six of these families displayed a familial inheritance pattern, whereas three families (T0197, T0198, and T0202) showed a de novo appearance in the proband.

The first four families described here exhibit novel frameshift mutations resulting in a truncated TRPS1 pro-
Table 1. Summary of TRPS1 mutations

\begin{tabular}{cllll}
\hline Family & Location & Mutation & Amino acid change & Syndrome \\
\hline T0142 & Exon 4 & 1403delA & Stop at codon 471 & TRPS I \\
T0139 & Exon 4 & 1449delG & Stop at codon 486 & TRPS I \\
T0197 & Exon 4 & C1591T & R531X & TRPS I \\
T0201 & Exon 4 & 1913insG & Stop at codon 672 & TRPS I \\
T0150 & Exon 4 & C2047T & R683X & TRPS I \\
T0199 & Exon 5 & C2518T & R840X & TRPS I \\
T0153 & Exon 6 & G2723A & R908Q & TRPS III \\
T0202 & Exon 6 & C2756T & A919V & TRPS III \\
T0198 & Exon 7 & 3159-60delAT & Stop at codon 1057 & TRPS I \\
\hline
\end{tabular}

tein. Family T0139 displays a novel deletion of a guanine in exon 4 at position 1449. This frameshift results in a premature stop codon at amino acid 486 (Fig. 1a, Table 1). Family T0198 shows another novel, de novo mutation in exon 7 at position 3159-3160. The 2-bp deletion of adenine and thymine also results in a truncated protein because of a premature stop codon at amino acid 1057 (Fig. 1b, Table 1). A single nucleotide deletion of an adenine at position 1403 on exon 4 is seen in family T0142. The mutation in this family also causes a frameshift, leading to a premature stop codon at amino acid 471 (Fig. 1c, Table 1). Finally, family T0201 displays an insertion of a guanine at nucleotide position 1913 on exon 4. This particular mutation again produces a truncated TRPS1 protein because of the premature stop codon at amino acid 672 within the fifth zinc finger (Fig. 1d, Table 1).

The final three TRPS I families presented here all show recurrent mutations (Momeni et al. 2000; Lüdecke et al. 2001). Family T0150 shows a transition of cytosine to thymine at position 2047 on exon 4, which causes the amino acid change R683X, also interrupting the fifth of nine zinc fingers in the TRPS1 protein (Fig. 1e, Table 1). Another cytosine-to-thymine transition is found in family T0199. This mutation occurs at position 2518 on exon 5, also causing a premature stop with the amino acid change R840X (Fig. 1f, Table 1). Although this particular mutation does not result in a robust peak in Fig. 1, there is a strong difference between the electropherograms of the affected and unaffected individuals within this family. These differences are seen independent of which strand was sequenced. Also, this particular phenomenon is not isolated to just this individual or family. Momeni et al. (2000) demonstrated another family that displayed a similar weak signal for this recurrent mutation. Finally, family T0197 shows another cytosine-tothymine transition. This de novo mutation is located at position 1591 on exon 4, causing a premature stop codon with the amino acid substitution R531X (Fig. 1g, Table 1).

The final two mutations described here are missense mutations in the GATA DNA-binding zinc finger believed to cause TRPS III. Family T0153 displays a recurrent missense mutation of a guanine to adenine occurring at position 2723 on exon 6. This particular mutation produces the amino acid substitution R908Q (Fig. 1h, Table 1). The de novo, missense mutation of cytosine to thymine at nucleotide position 2756 is seen in family T0202. This mutation causes the amino acid substitution A919V (Fig. i, Table 1). Although this mutation only results in a minor amino acid 
Fig. 1a-i. Pedigrees for families showing mutations in the TRPS1 gene. An electropherogram and subsequent sequence accompany each of the pedigrees. The mutated sequence is shown below the normal TRPS1 sequence (bold letters show exact location of each mutation). An asterisk denotes affected individuals in each family available for analysis. a Family T0139: guanine deletion; b Family T0198: adenine-thymine deletion; c Family T0142: adenine deletion; d Family T0201: guanine insertion; e Family T0150: cytosine-to-thymine transition; f Family T0199: cytosineto-thymine transition; g Family T0197: cytosine-to-thymine transition; h Family T0153: guanineto-adenine transition; i Family T0202: cytosine-to-thymine transition. Summary of specific mutations is shown in Table 1 a)
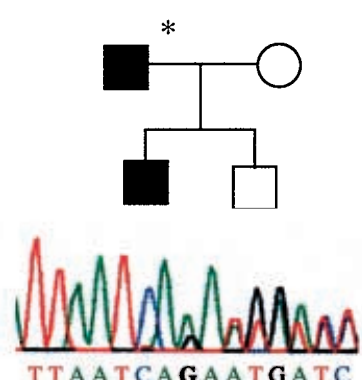

d)
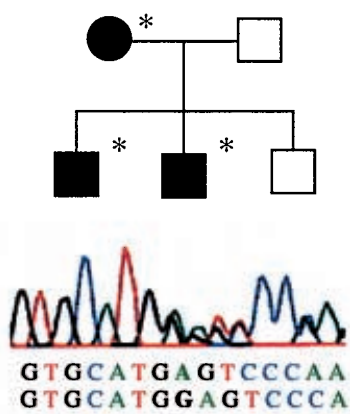

g)
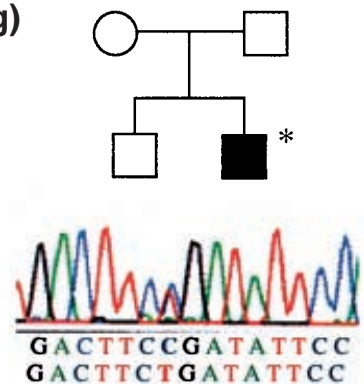

b)
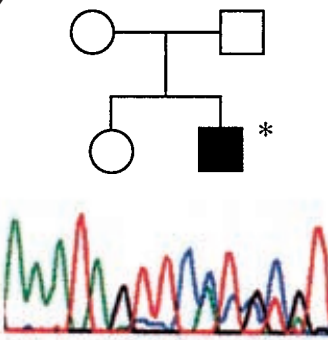

A A A T A G T T CA T C C G T A A A T AGTTCCCGTAT

e)
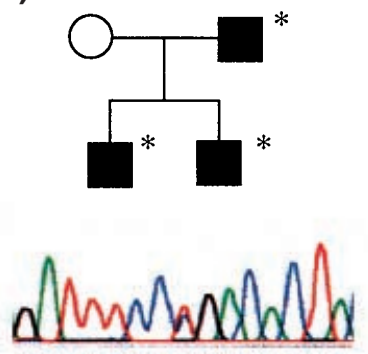

GATT TCCCGACACTA GATT TCCTGACACTA

h)
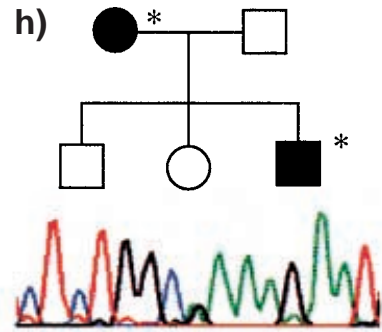

C T C TGGCGAAA GAAT C T C TGGCAAAA GAAT c)
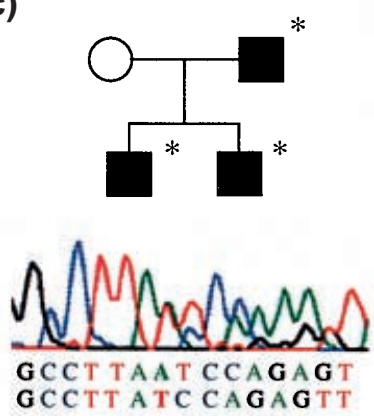

f)
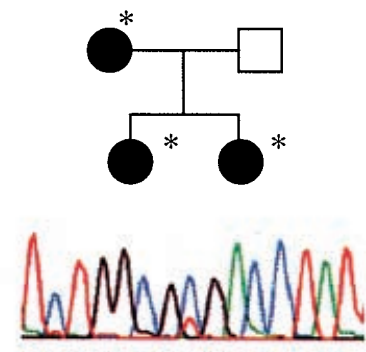

T CT GGCGCGACCTAT TCTGGCGTGACCTAT

i)
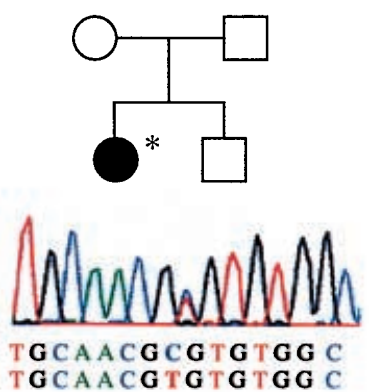

change, it occurs in a critical and conserved region of the protein. It also represents a de novo mutation because neither parent's DNA shows the mutation.

\section{Discussion}

TRPS is believed to result from a haploinsufficiency of the TRPS1 gene. The subsequent reduction in protein concentration or activity can result from either specific base mutations within the TRPS1 gene (as shown here), or by the deletion of the entire gene, as is common in all TRPS II (LGS) and some TRPS I patients. Each of the frameshift and nonsense mutations identified here is predicted to result in a truncated, nonfunctional TRPS1 protein. In six of the nine families (T0142, T0139, T0197, T0150, T0199, and T0201), nonsense or frameshift mutations occur prior to the nuclear localization signals (NLS). Therefore, incorrect protein localization may be responsible for their loss of function. Although the frameshift mutation in family T0198

resides after the NLS, the resultant protein lacks the tandem Ikaros-like zinc fingers located near the $\mathrm{C}$ terminus. Previous studies with the Ikaros family of transcription factors have demonstrated that the tandem zinc fingers are necessary for homo- and hetero-dimerization, and for normal transcription factor activity (Sun et al. 1999). It has been suggested that the TRPS1 protein also might selfassociate, or hetero-dimerize with other Ikaros-like transcription factors to maintain its own normal function (Lüdecke et al. 2001). Although the Ikaros family of transcription factors is primarily expressed in lymphoid and erythroid cells, two new members, Eos and Pegasus, have been shown to be expressed in many of the same tissues (e.g., brain, heart, lung, kidney, and skeletal muscle) as TRPS1 (Perdomo et al. 2000). Taken together, this suggests that the transcription factors Eos and Pegasus could potentially interact with the TRPS1 protein.

Missense mutations located in the GATA zinc finger have also been identified in families T0153 and T0202. Family T0153 displays a recurrent mutation that presumably affects the DNA binding affinity of the GATA zinc-finger 
by substituting a neutral, polar (Q) amino acid for a basic (R) amino acid near the apex of the zinc-finger domain. In accordance with observations made by Lüdecke et al. (2001), family T0153 also exhibits characteristics indicative of TRPS III because of the missense mutation in the GATA zinc finger. Family T0202 also exhibits a de novo missense mutation that potentially disrupts the GATA zinc finger at amino acid position 919. The slight amino acid change from a nonpolar, hydrophobic amino acid (A) to another nonpolar, hydrophobic amino acid (V) occurs in a crucial zincbinding domain between the second group of cysteines (CNA/VC) in the GATA zinc-finger motif. This amino acid position is a site of recurrent mutations, although this potential mutation would be the most subtle mutation recorded to date. This particular alanine is also evolutionarily conserved in all mammalian GATA and GATA-like zinc fingers, suggesting its importance in normal GATA zincfinger structure and function.

Acknowledgments This research was supported by a grant from the National Institutes of Health to D.E.W. (HD27981).

\section{References}

Hou J, Parrish J, Lüdecke H-J, Sapru M, Wang Y, Chen W, Hill A, Siegel-Bartlet J, Northrup H, Elder FFB, Chinault C, Horsthemke B, Wagner MJ, Wells DE (1995) A 4-megabase YAC contig that spans the Langer-Giedion syndrome region on human chromosome 8q24.1: use in refining the location of the trichorhinophalangeal syndrome and multiple exostoses genes (TRPS1 and EXT1). Genomics 29:87-97

Lüdecke H-J, Wagner MJ, Nardmann J, La Pillo B, Parrish JE, Willems PJ, Haan EA, Frydman M, Hamers GJH, Wells DE, Horsthemke B (1995) Molecular dissection of a contiguous gene syndrome: localization of the genes involved in Langer-Giedion syndrome. Hum Mol Genet 4:31-36

Lüdecke H-J, Schaper J, Meinecke P, Momeni P, Gross S, von Holtum D, Hirche H, Abramowicz MJ, Albrecht B, Apacik C, Christen H-J, Claussen U, Devriendt K, Fastnacht E, Forderer A, Friedrich U, Goodship THJ, Greiwe M, Hamm H, Hennekam RCM, Hinkel GK, Hoeltzenbein M, Kayserili H, Majewski F, Mathieu M, McLeod R, Midro AT, Moog U, Nagai T, Niikawa N, Ørstavik KH, Plöchl E, Seitz C, Schmidtke J, Tranebjaerg L, Tsukahara M, Wittwer B, Zabel B, Gillessen-Kaesbach G, Horsthemke B (2001) Genotypic and phenotypic spectrum in tricho-rhino-phalangeal syndrome types I and III. Am J Hum Genet 68:81-91

Momeni P, Glöckner G, Schmidt O, von Holtum D, Albrecht B, Gillessen-Kaesbach G, Hennekam R, Meinecke P, Zabel B, Rosenthal A, Horsthemke B, Lüdecke H-J (2000) Mutations in a new gene, encoding a zinc-finger protein, cause tricho-rhinophalangeal syndrome type I. Nat Genet 24:71-74

Niikawa N, Kamei T (1986) The Sugio-Kajii syndrome, proposed tricho-rhino-phalangeal syndrome type III. Am J Med Genet 24:759-760

Perdomo J, Holmes M, Chong B, Crossley M (2000) Eos and Pegasus, two members of the ikaros family of proteins with distinct DNA binding activities. J Biol Chem 49:38347-38354

Sun L, Heerema N, Crotty L, Wu X, Navara C, Vassilev A, Sensel M, Reaman GH, Uckun FM (1999) Expression of dominant-negative and mutant isoforms of the antileukemic transcription factor Ikaros in infant acute lymphoblastic leukemia. Proc Natl Acad Sci USA 96:680-685 